\title{
Identification and characterization of an efficient acyl-CoA: diacylglycerol acyltransferase 1 (DGAT1) gene from the microalga Chlorella ellipsoidea
}

Xuejie Guo ${ }^{1,2}$, Chengming Fan ${ }^{1}$, Yuhong Chen ${ }^{1}$, Jingqiao Wang ${ }^{3}$, Weibo Yin ${ }^{1}$, Richard R. C. Wang ${ }^{4}$ and Zanmin $\mathrm{Hu}^{1,2,5^{*}}$

\begin{abstract}
Background: Oil in the form of triacylglycerols (TAGs) is quantitatively the most important storage form of energy for eukaryotic cells. Diacylglycerol acyltransferase (DGAT) is considered the rate-limiting enzyme for TAG accumulation. Chlorella, a unicellular eukaryotic green alga, has attracted much attention as a potential feedstock for renewable energy production. However, the function of DGAT1 in Chlorella has not been reported.

Results: A full-length cDNA encoding a putative diacylglycerol acyltransferase 1 (DGAT1, EC 2.3.1.20) was obtained from Chlorella ellipsoidea. The 2,142 bp open reading frame of this CDNA, designated CeDGAT1, encodes a protein of 713 amino acids showing no more than 40\% identity with DGAT1s of higher plants. Transcript analysis showed that the expression level of CeDGAT1 markedly increased under nitrogen starvation, which led to significant triacylglycerol (TAG) accumulation. CeDGAT1 activity was confirmed in the yeast quadruple mutant strain $\mathrm{H} 1246$ by restoring its ability to produce TAG. Upon expression of CeDGAT1, the total fatty acid content in wild-type yeast (INVSc1) increased by $142 \%$, significantly higher than that transformed with DGAT1s from higher plants, including even the oil crop soybean. The over-expression of CeDGAT1 under the NOS promoter in wild-type Arabidopsis thaliana and Brassica napus var. Westar significantly increased the oil content by $8-37 \%$ and $12-18 \%$ and the average 1,000 -seed weight by 9-15\% and 6-29\%, respectively, but did not alter the fatty acid composition of the seed oil. The net increase in the 1,000-seed total lipid content was up to $25-50 \%$ in both transgenic Arabidopsis and B. napus.
\end{abstract}

Conclusions: We identified a gene encoding DGAT1 in C. ellipsoidea and confirmed that it plays an important role in TAG accumulation. This is the first functional analysis of DGAT1 in Chlorella. This information is important for understanding lipid synthesis and accumulation in Chlorella and for genetic engineering to enhance oil production in microalgae and oil plants.

Keywords: Chlorella ellipsoidea, Diacylglycerol acyltransferase, Nitrogen starvation, Seed oil content, Seed weight, Triacylglycerol

\footnotetext{
* Correspondence: zmhu@genetics.ac.cn

${ }^{1}$ Institute of Genetics and Developmental Biology, Chinese Academy of Sciences, Beijing 100101, China

${ }^{2}$ University of Chinese Academy of Sciences, Beijing 100049, China

Full list of author information is available at the end of the article
} 


\section{Background}

Triacylglycerols (TAGs) are the main storage lipids in various organisms, such as oilseed plants, oleaginous fungi, yeasts, and microalgae. They are also a major source of highly reduced carbon molecules for food and fuel $[1,2]$. TAGs are synthesized in endoplasmic reticulum (ER) and accumulate as oil droplets in lipid bodies, which are generated by budding off from the outer ER membrane [3, 4]. In the Kennedy pathway, TAGs are synthesized by sequentially adding acyl-CoAs to the $s n-1, s n-2$ and $s n-3$ positions of a glycerol-3-phosphate molecule [5], which is controlled by four important enzymes, glycerol-3-phosphate acyltransferase (GPAT; EC 2.3.1.15), lyso-phosphatidic acid acyltransferase (LPAT; EC 2.3.1.51), phosphatidate phosphatase (PAP; EC 3.1.3.4) and diacylglycerol acyltransferase (DGAT; EC 3.2.1.20) [6]. DGAT has been proposed to be the rate-limiting enzyme for TAG accumulation [7, 8].

In eukaryotes, three types of DGATs have been reported: the endoplasmic reticulum (ER)- localized DGAT1, DGAT2 and the soluble cytosolic DGAT3. Among them, DGAT1 and DGAT2 are responsible for the bulk of TAG synthesis in most organisms [9]. It has been proposed that these two enzymes have no redundant functions in TAG biosynthesis [10]. DGAT1 plays a dominating role in the determination of oil accumulation and fatty acid composition in seed oils [6], and DGAT2 may influence the content and composition of some plant seed oils containing unusual fatty acids (e.g., epoxy and hydroxyl) [11-13]. The role of the cytosolic DGAT3 has not yet been determined.

DGAT1s are ER membrane-bound proteins and possess six to nine transmembrane domains [14]. The most variable region of DGAT1 is the hydrophilic $\mathrm{N}$ terminus, which is quite unique for each DGAT1 and might serve distinct functions in different organisms [15]. Several conserved motifs, including acyl-CoA binding motif, DAG binding motif, the fatty acid-binding protein signature and a putative C-terminal ER retrieval motif, have been identified in DGAT1 [16]. Recently, site-directed mutagenesis was used to demonstrate the importance of some conserved residues in DGAT1s. For instance, mutagenesis at P216 and F439 in Tropaeolum majus DGAT1 resulted in a total loss of DGAT1 activity, while the substitution of S197 with alanine in a putative SnRK1 target site resulted in a strong increase in DGAT1 activity in the range of $38 \%$ to $80 \%$ [6].

The first eukaryotic DGAT1 gene was cloned from mouse [17], followed by isolation from other organisms $[10,16,18-26]$. Many studies have investigated DGAT1s because of their important roles in TAG synthesis and have tried to use them to alter the quality and quantity of storage lipids in higher plants. For instance, the AS11 mutant of Arabidopsis, having reduced DGAT activity, showed a $75 \%$ reduction in seed lipids, but the expression of Arabidopsis DGAT1 in the AS11 mutant restored the wild-type levels of TAG and very-long-chain fatty acid content [27]. Moreover, the over-expression of $A t D$ GAT1 can greatly enhance the TAG content of transformed tobacco $[19,28]$. Subsequently, Tropaeolum majus DGAT1 significantly contributes to seed oil biosynthesis in wild-type Arabidopsis and Brassica napus by over-expression [6]. The co-expression of an epoxygenase from Stokesia laevis, SlEPX, and VgDGAT1 or VgDGAT2 from Vernonia galamensis greatly increased the accumulation of vernolic acid in both petunia leaves and soybean somatic embryos [13]. The over-expression of DGAT1 from Jatropha curcas showed an enhanced total oil content in seeds but did not show any phenotypic differences [25].

Unlike oil crops, microalgae have higher biomass production rates and many are exceedingly rich in oil. Therefore, microalgae have been regarded as potential resources for producing biodiesel, especially neutral lipids (e.g., triacyglycerols; TAGs) [29-31]. Many microalgal strains have the ability to accumulate substantial amounts of lipids in the form of TAGs under stress conditions, such as nitrogen starvation [30]. So far, several $D G A T$ s have been cloned and functionally characterized from microalgae. For instance, DGAT1-like [16] and DGAT2B [32] from the diatom Phaeodactylum tricornutum have been functionally characterized in a TAGdeficient mutant in the yeast Saccharomyces cerevisiae. Furthermore, the over-expression of PtDGAT2 in P. tricornutum resulted in a $35 \%$ increase in the neutral lipid content, and the fatty acid composition showed a significant increase in the proportion of polyunsaturated fatty acids [33]. Two DGAT2s (OtDGAT2A and OtDGAT2B) have been identified and characterized from Ostreococcus tauri, and OtDGAT2B possesses broad substrate specificity [34]. TpDGAT2 from the marine diatom Thalassiosira pseudonana significantly affects the fatty acid profile of TAG [35]. In Chlamydomonas reinhardtii, homology searches identified five DGAT2, encoded by DGTT1-DGTT5 [36]. Among them, DGTT1 and DGTT3 are active in TAG synthesis following nitrogen deprivation [37]. The expression of CrDGTT2 in Arabidopsis increased the leaf TAG content, with some molecular species containing very-long-chain fatty acids [38]. A gene encoding DGAT1 was also identified in $C$. reinhardtii after the transcript-based correction of gene models [39]. Other putative DGAT genes have been annotated in the genomes of some microalgae, such as Chlorella variabilis, Coccomyxa sp. C-169, Volvox carteri f. nagariensis, Ostreococcus lucimarinus, Fragilariopsis cylindrus and so on [40]. However, to date, there are few reports on the function of DGAT from the unicellular eukaryotic green alga, Chlorella, which is a desirable resource for producing biodiesel. Therefore, research on DGAT from Chlorella will advance our understanding of 
the molecular mechanisms underlying lipid metabolism during oil accumulation and will also provide a new means to improve the oil quality and content of microalgae and oil crops.

In the present study, we isolated a DGAT1 gene $(C e D$ GAT1) from C. ellipsoidea, a unicellular eukaryotic green alga that can be easily cultured under either autotrophic or heterotrophic conditions, and characterized its function in yeast and higher plants (Arabidopsis and B. napus). Compared with DGAT1s of higher plants, such as Glycine max, Arabidopsis and Brassica oleracea, CeDGAT1 could more effectively enhance fatty acid accumulation in the wild-type yeast (INVSc1). The over-expression of $\mathrm{CeD}$ GAT1 can significantly enhance the seed oil content and seed weight in Arabidopsis and B. napus. Furthermore, the expression pattern of the isolated DGAT1 gene was investigated. This study would be helpful for understanding the function of DGAT from microalgae and for improving oil production in B. napus.

\section{Results \\ Identification, sequence and phylogenetic analysis of CeDGAT1 in C. ellipsoidea}

Based on the expressed sequence tag (EST) data of $C$. ellipsoidea, a full-length cDNA fragment of C. ellipsoidea DGAT1, designated as CeDGAT1, was cloned and identified. The nucleotide sequence has a full CDS of 2,142 bp, encoding a polypeptide of 713 amino acid residues with a calculated molecular mass of $81.76 \mathrm{kDa}$. It was registered in GenBank (ID No. KT779429). CeDGAT1 shared no more than $40 \%$ identity with DGAT1s of higher plants, such as G. $\max (40 \%), Z$. mays (39\%), R. communis (38\%), Arabidopsis (38\%), B. napus (36\%), $V$. fordii (35\%) and J. curcas (34\%).

To examine the relationships among different sources of DGAT, a phylogenetic tree was generated from an alignment of the deduced amino acid sequences of CeDGAT1 with 44 DGAT homologues from other species (including membrane-bound DGAT1 and DGAT2 and cytosolic DGAT3). The dendrogram grouped CeDGAT1 with members of the DGAT1 family. DGAT2 and DGAT3 formed a separate cluster different from DGAT1 branches (Fig. 1). In detail, CeDGAT1 clustered together with APDGAT1 (Auxenochlorella protothecoides) and CvDGAT1 (Chlorella vulgaris) and formed a distinct lineage. Furthermore, CeDGAT1 is more closely related to DGAT1s from animals and fungi than to DGAT1s from plants.

Protein analysis with the TMpred program [41] predicted nine strongly hydrophobic transmembrane regions, which is consistent with the nine transmembrane domains predicted for $B$. napus DGAT1, T. majus DGAT1 and Arabidopsis DGAT1 [6, 9, 20]. Using the PROSITE database [42], a number of putative functional motifs, including $N$-glycosylation, cAMP- and cGMPdependent protein kinase phosphorylation, protein kinase C phosphorylation, casein kinase II phosphorylation, tyrosine kinase phosphorylation, $N$-myristoylation and amidation sites were identified in CeDGAT1 (Additional file 1: Table S1). Compared with AtDGAT1, $N$-glycosylation and amidation sites were found only in CeDGAT1, while a leucine zipper pattern was detected only in AtDGAT1. It remains to be determined whether these sites are important for the functional regulation of the enzyme in vivo.

As previously reported [43, 44], a consensus amino acid sequence for an acyl-CoA binding motif (Fig. 2, I) and a conserved sequence for the DAG-binding motif (Fig. 2, III) of DGAT were also found in CeDGAT1. There was also a fatty acid-binding protein signature spanning residues Ala571 to Asn587 (Fig. 2, II) containing a putative tyrosine phosphorylation site: Tyr582 [6]. The CeDGAT1 protein contained an invariant proline (Pro381), which is thought to participate in presenting the fatty acyl group to the active site for esterification to (diacyl) glycerol and is critical for DGAT1 activity [45]. A highly conserved serine residue (Ser410) was essential for the activity of acyl-CoA: cholesterol acyltransferase, an enzyme closely related to DGAT1 [24, 46]. CeDGAT1 showed a leucine zipper motif with only one conserved leucine (Leu386) in the sequence. A visual examination of CeDGAT1 also revealed the sequence of a putative $\mathrm{C}$-terminal ER retrieval motif (YYHDW, Fig. 2, IV), which is similar to other DGAT1 proteins in plants [11].

\section{Lipid analysis and expression pattern of CeDGAT1 in C. ellipsoidea}

To understand the relationship between CeDGAT1 expression and lipid synthesis, we investigated the expression pattern of CeDGAT1, the variation in biomass, and the total fatty acid (TFA) and TAG contents in heterotrophic cultures of $C$. ellipsoidea on nitrogen-replete and nitrogen-depleted $(1 / 4 \mathrm{~N})$ media (Fig. 3). As shown in Fig. 3a, the algal cell biomass increased more slowly in nitrogen-depleted cultures than in nitrogen-replete cultures, but both the TFA content and TAG content increased rapidly from $19 \%$ and $9 \%$ to $44 \%$ and $23 \%$, respectively, from 36 to $108 \mathrm{~h}$ (Fig. $3 \mathrm{~b}$ and c). On the contrary, TFA and TAG contents were not significantly changed in the growth process of nitrogen sufficient condition. So there was a $108 \%$ and $212 \%$ increase in TFA content and TAG content, respectively at the $108^{\text {th }}$ hour under nitrogen-depleted culture condition than under nitrogen sufficient culture condition.

Quantitative real-time PCR was performed to examine the expression profiles of CeDGAT1 in C. ellipsoidea cells under nitrogen-replete and nitrogen-depleted (1/ $4 \mathrm{~N}$ ) conditions (Fig. 3d). 18S rRNA was used as an internal reference control. We noted that CeDGAT1, 


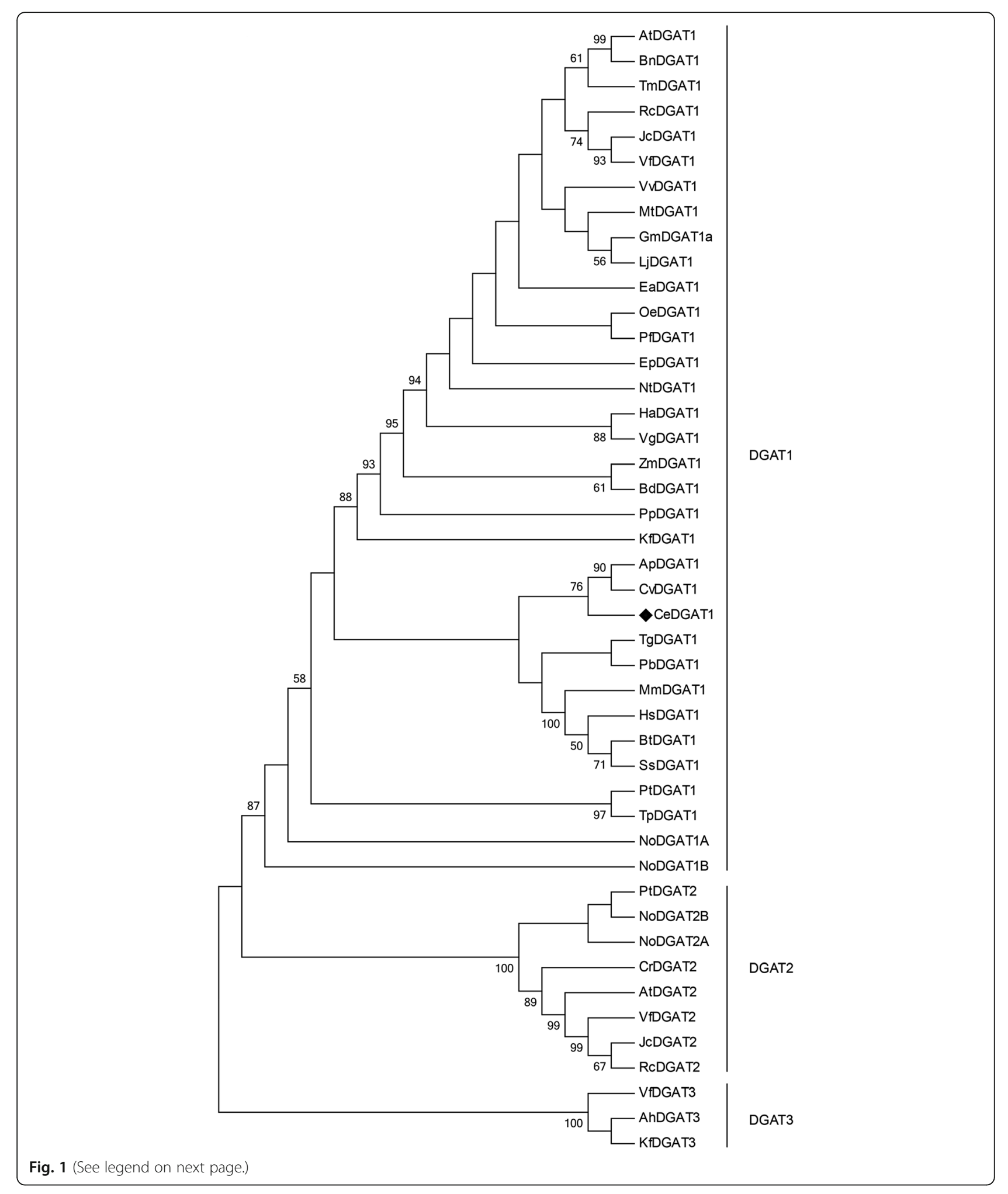




\begin{abstract}
(See figure on previous page.)
Fig. 1 Phylogenetic relationship between CeDGAT1 and DGATs from plants, animals, fungi and microalgae. Sequence alignments were generated with MAFFT, and the phylogenetic tree was constructed by using the the maximum likelihood (ML) method. AtDGAT1 and AtDGAT2 (Arabidopsis thaliana, accession no. CAB45373.1 and NP_566952.1, respectively); BnDGAT1 (Brassica napus, accession no. AAF64065.1); TmDGAT1 (Tropaeolum majus, accession no. AAM03340.2); RcDGAT1 and RcDGAT2 (Ricinus communis, accession no. AAR11479.1 and XP_002528531.1, respectively); JcDGAT1 and JCDGAT2 (Jatropha curcas, accession no. ABB84383.1 and AFV61670.1); VfDGAT1, VfDGAT2 and VfDGAT3 (Vernicia fordii, accession no. ABC94471.1, ABC94473.1 and AGL81309.1, respectively); VvDGAT1 (Vitis vinifera, accession no. XP_002279345.1); MtDGAT1 (Medicago truncatula, accession no. XP_003595231.1); GmDGAT1a (Glycine max, accession no. BAE93460.1); LjDGAT1 (Lotus japonicas, accession no. AAW51456.1); EaDGAT1 (Euonymus alatus, accession no. AAV31083.1); OeDGAT1 (Olea europaea, accession no. AAS01606.1); PfDGAT1 (Perilla frutescens, accession no. AAG23696.1); EpDGAT1 (Echium pitardii, accession no. ACO55634.1); NtDGAT1 (Nicotiana tabacum, accession no. AAF19345.1); HaDGAT1 (Helianthus annuus, accession no. ACD67882.1); VgDGAT1 (Vernonia galamensis, accession no. ABV21945.1); ZmDGAT1 (Zea mays, accession no. ABV91586.1); BdDGAT1 (Brachypodium distachyon, accession no. XP_003568769.1); PpDGAT1 (Physcomitrella patens, accession no. XP_001770929.1); KfDGAT1 and KfDGAT3 (Klebsormidium flaccidum, accession no. GAQ91878.1 and GAQ88368.1, respectively); APDGAT1 (Auxenochlorella protothecoides, accession no. XP_011402032.1); CVDGAT1 (Chlorella vulgaris, accession no. ALP13863.1); TgDGAT1 (Toxoplasma gondii, accession no. AAP94209.1); PbDGAT1 (Paracoccidioides brasiliensis, accession no. EEH17170.1); MmDGAT1 (Mus musculus, accession no. NP_034176.1); HsDGAT1 (Homo sapiens, accession no. NP_036211.2); BtDGAT1 (Bos Taurus, accession no. AAL49962.1); SsDGAT1 (Sus scrofa, accession no. NP_999216.1); PtDGAT1 and PtDGAT2 (Phaeodactylum tricornutum, accession no. ADY76581.1 and AFM37314.1, respectively); TpDGAT1 (Thalassiosira pseudonana, accession no. XP_002287215.1); NoDGAT1A, NoDGAT1B, NoDGAT2A and NoDGAT2B (Nannochloropsis oceanica) [55]; CrDGAT2 (Chlamydomonas reinhardtii, accession no. XP_001693189.1), and AhDGAT3 (Arachis hypogaea, accession no. AAX62735.1)
\end{abstract}

which catalyses the last committed step in TAG biosynthesis, was downregulated under nitrogen-replete conditions. Nevertheless, CeDGAT1 showed transient upregulation, with its transcript level peaking at $84 \mathrm{~h}$ following the onset of nitrogen depletion and declining thereafter. The upregulation of CeDGAT1 was concomitant with the increase in the TFA and TAG contents under nitrogen deprivation, suggesting that CeDGAT1 was highly induced by nitrogen deprivation and that its increased expression coupled with lipid content change may play an important role in TAG accumulation.

\section{CeDGAT1 can recover the TAG synthesis of the quadruple mutant yeast strain $\mathrm{H} 1246$}

To verify the diacylglycerol acyltransferase activity of CeDGAT1, the CeDGAT1 gene was heterologously expressed in the TAG-deficient S. cerevisiae quadruple mutant strain H1246 [47], which lacks the four genes DGA1, LRO1, ARE1 and ARE2 encoding DGAT, PDAT (phosphatidylcholine: diacylglycerol acyltransferase), ASAT1 (acyl-CoA: sterol acyltransferase 1) and ASAT2 (acyl-CoA: sterol acyltransferase 2), respectively. These four genes are essential for the formation of neutral lipids. Lipid bodies can be formed by the expression of at least one of four genes. INVSc1 and H1246 cells harbouring an empty pYES2.0 vector were used as positive and negative controls, respectively.

Nile Red was used to stain lipid bodies in yeast cells. Lipid bodies were present in the wild-type yeast strain INVSc1 or the mutant strain transformed with the $\mathrm{CeD}$ GAT1 gene but were undetectable in the quadruple mutant strain carrying the empty expression vector (pYES2.0) (Fig. 4a). The total lipids were extracted from yeast cells and then subjected to TLC (Thin-Layer Chromatography) analysis. Upon expression of CeDGAT1, a prominent band corresponding to TAG appeared on the TLC plate as expected, whereas no TAGs were identified in mutant yeast cells lacking the endogenous yeast DGAT and PDAT activities (Fig. 4b), which is consistent with the results from Nile Red staining (Fig. 4a). These results suggest that CeDGAT1 can successfully restore the ability of the quadruple mutant strain H1246 to form neutral lipids and confirm that CeDGAT1 encodes a functional protein capable of catalysing the last step of TAG biosynthesis.

\section{Heterologous expression of CeDGAT1 can more significantly increase the total fatty acid content in the wild-type yeast than DGAT1s from some higher plants}

We separately transferred CeDGAT1 and another three $D G A T 1$ genes from higher plants, including oil crop $G$. max, $A$. thaliana and $B$. oleracea, into the wild-type yeast (INVSc1). RT-PCR results showed that the DGAT1 genes had nearly identical expression patterns in transgenic yeast (Additional file 2: Figure S1). The fatty acid contents of yeast carrying DGAT1 genes were measured by GC. As shown in Fig. 5, the total fatty acid contents of yeast significantly increased due to the expression of different DGAT1s when compared to the yeast transformed with pYES2.0. In detail, the total fatty acid content in yeast carrying AtDGAT1, GmDGAT1, BoDGAT1 and CeDGAT1 was $234.7 \mu \mathrm{g} / \mathrm{mg}, 243.3 \mu \mathrm{g} / \mathrm{mg}, 258.5 \mu \mathrm{g} /$ $\mathrm{mg}$ and $290.0 \mu \mathrm{g} / \mathrm{mg}$, respectively. Among the yeast expressing different DGAT1 genes, the total fatty acid content in the yeast transformed with CeDGAT1 increased most remarkably, by $142 \%$. In contrast (compared with the yeast expressing CeDGAT1), the total fatty acid contents of the yeasts transformed with the three DGAT1 genes from higher plants significantly decreased by $19 \%$ (AtDGAT1), 16\% (GmDGAT1) and 11\% (BoDGAT1), respectively. These results suggest that CeDGAT1 may 


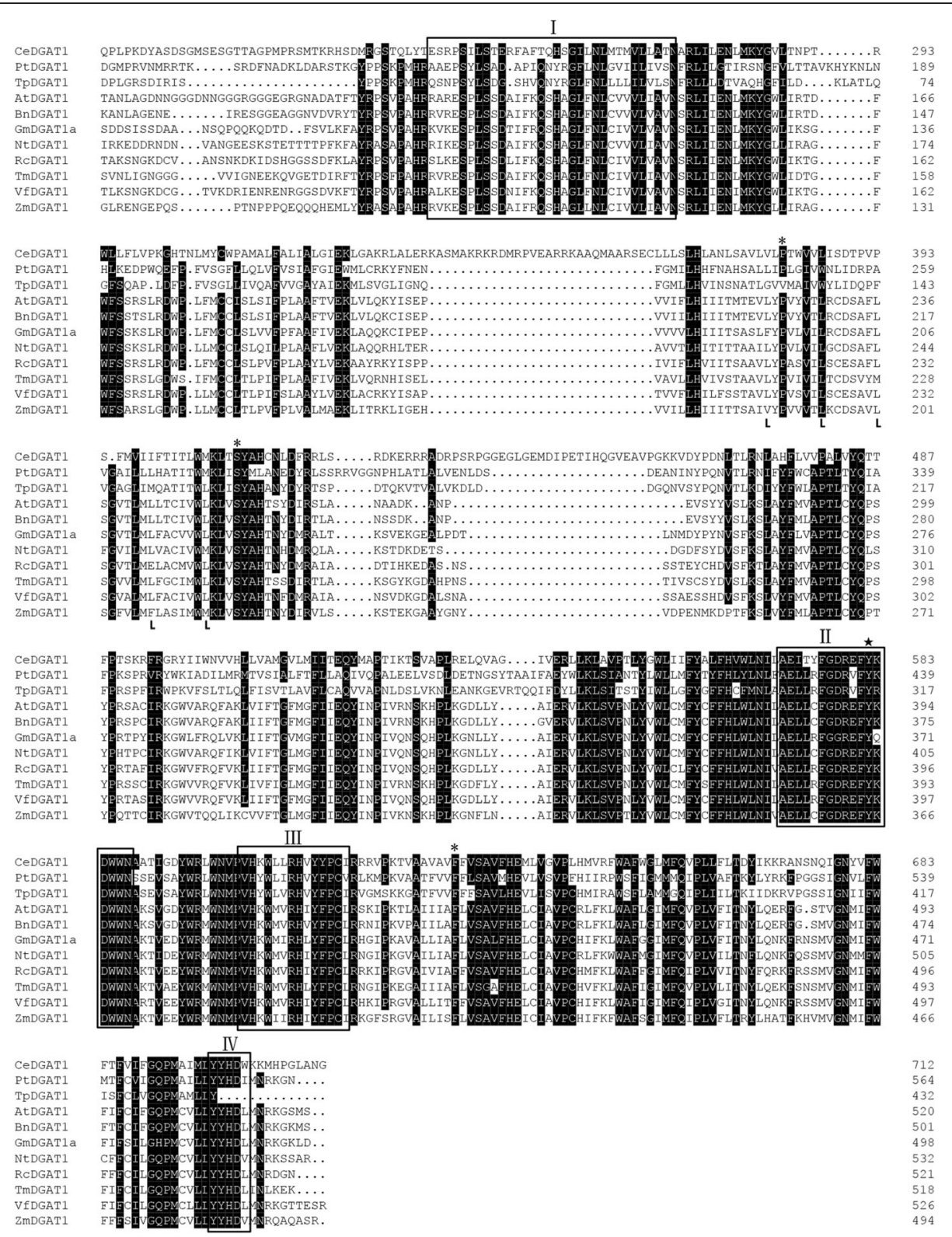

Fig. 2 Sequence comparison of CeDGAT1 with DGAT1s from other species. PtDGAT1 (Phaeodactylum tricornutum, accession no. ADY76581.1), TpDGAT1 (Thalassiosira pseudonana, accession no. XP_002287215.1), AtDGAT1 (Arabidopsis thaliana, accession no. CAB45373.1), BnDGAT1 (Brassica napus, accession no. AAF64065.1), GmDGAT1a (Glycine max, accession no. BAE93460.1), NtDGAT1 (Nicotiana tabacum, accession no. AAF19345.1), RcDGAT1 (Ricinus communis, accession no. AAR1 1479.1), TmDGAT1 (Tropaeolum majus, accession no. AAM03340.2), VfDGAT1 (Vernicia fordii, accession no. ABC94471.1), and ZmDGAT1 (Zea mays, accession no. ABV91586.1). Identical amino acid residues are highlighted in black. Conserved motifs or putative signatures are boxed, such as the acyl-CoA binding signature (I); the fatty acid protein signature (II), which contains a tyrosine phosphorylation site ( $\star$ ); the DAG-binding site (III); and the putative endoplasmic reticulum retrieval motif in the C-terminus (IV). The highly conserved proline and serine residues are marked by asterisks. The region containing a conserved leucine repeat $(L)$ is also marked

function in improving the oil content of plants, especially oil crops.

\section{Over-expression of CeDGAT1 enhances the seed oil} content and seed weight in higher plants

To explore CeDGAT1 as a tool to manipulate acyl-CoA pools and to engineer TAGs in plants, CeDGAT1 was over-expressed in Arabidopsis and B. napus var. Westar under the control of the constitutive NOS promoter. In Arabidopsis, three independent homozygous lines were selected for advancement to the T4 generation and used for detailed analysis. RT-PCR results showed that the CeDGAT1 transcript was expressed in transgenic lines over-expressing CeDGAT1 (Additional file 3: Figure S2). 

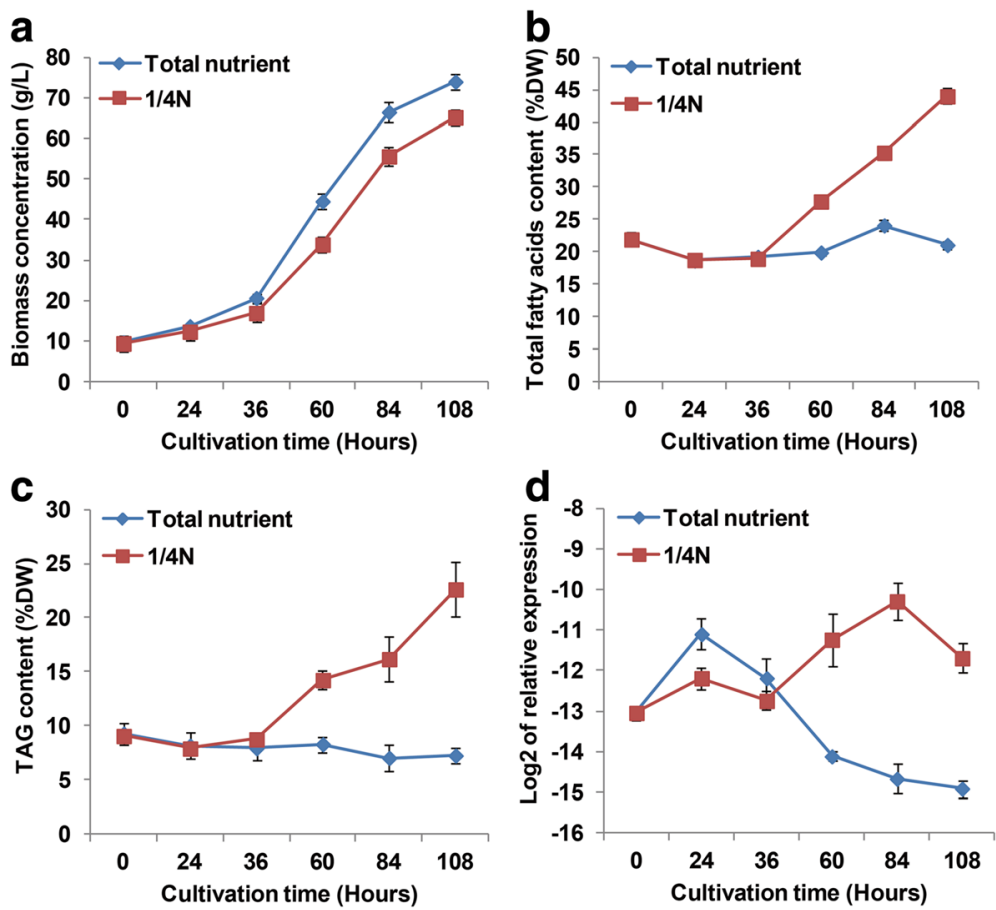

Fig. 3 Biomass concentration (a), TFA content (b), TAG content (c) and expression of CeDGAT1 (d) measured from 0 to $108 \mathrm{~h}$ in C. ellipsoidea cultures grown on nitrogen-replete or nitrogen-depleted $(1 / 4 \mathrm{~N})$ medium. The results are expressed as the mean \pm standard deviation $(n=3)$

The transgenic lines did not show any visible morphological difference from untransformed control plants (data not shown). GC analysis revealed that the transformation of wild-type Arabidopsis with CeDGAT1 leads to a higher seed oil content (Fig. 6a). The average total fatty acid content in wild-type Arabidopsis seeds was $31.5 \mathrm{mg} / 100 \mathrm{mg}$ of seed, but increased to $33.9-43.1 \mathrm{mg} /$ $100 \mathrm{mg}$ in the transgenic lines. Thus, the transgenic seeds displayed an approximately $8-37 \%$ higher oil content than that of wild-type plants. In addition, the 1,000seed weight of transgenic plants was 9-15\% greater than that of the control (Fig. 6b).

In $B$. napus, twelve transgenic plants were identified by PCR (Additional file 4: Figure S3), and four of the twelve independent T3 transgenic $B$. napus lines were chosen for further analysis. The RT-PCR results showed that the CeDGAT1 transcript was expressed in transgenic lines (Additional file 3: Figure S2). Again, transgenic $B$. napus lines did not show any visible morphological difference from wild-type plants (data not shown). The fatty acid content in the seeds of wild-type B. napus and transgenic lines was measured using GC. As shown in Fig. 7a, the average total fatty acid content in wild-type $B$. napus seeds was $39.6 \mathrm{mg} / 100 \mathrm{mg}$ of seed. In lines expressing CeDGAT1 under the NOS promoter, the total fatty acid content increased to $44.5-46.8 \mathrm{mg} / 100 \mathrm{mg}$ of seed, representing an increase of $12-18 \%$ over the control. Moreover, the average 1,000-seed weight in the CeDGAT1 transgenic lines increased by $6-29 \%$ compared to that of wild-type plants (Fig. 7b). These results indicated that the CeDGAT1 gene can stimulate fatty acid biosynthesis and enhance seed weight.

\section{Subcellular localization of CeDGAT1}

EGFP-tagged CeDGAT1 was expressed in tobacco BY-2 suspension cells and then examined under confocal laserscanning microscopy. As shown in Fig. 8, the distribution pattern of EGFP-CeDGAT1 was similar to that of endogenous ER stained with ER-tracker ${ }^{\text {Tw }}$ Red, and the signal was visualized around the nucleus and thread-like ER networks, indicating typical ER localization.

\section{Discussion}

TAGs are quantitatively the most important storage form of energy for eukaryotic cells. The synthesis of TAG from DAG by DGAT is believed to be the major flux control step in oil biosynthesis. Much research has focused on DGAT because it is an enzyme unique to TAG synthesis in plants. However, the function of DGAT1 from Chlorella has not been reported.

In this study we cloned and characterized a novel DGAT1 gene (CeDGAT1) from C. ellipsoidea. Proteinprotein BLAST showed that CeDGAT1 shared no more than $40 \%$ identity with DGAT1s of higher plants, which resulted in a difference in the predicted three-dimensional structures (Additional file 5: Figure S4). Functional 


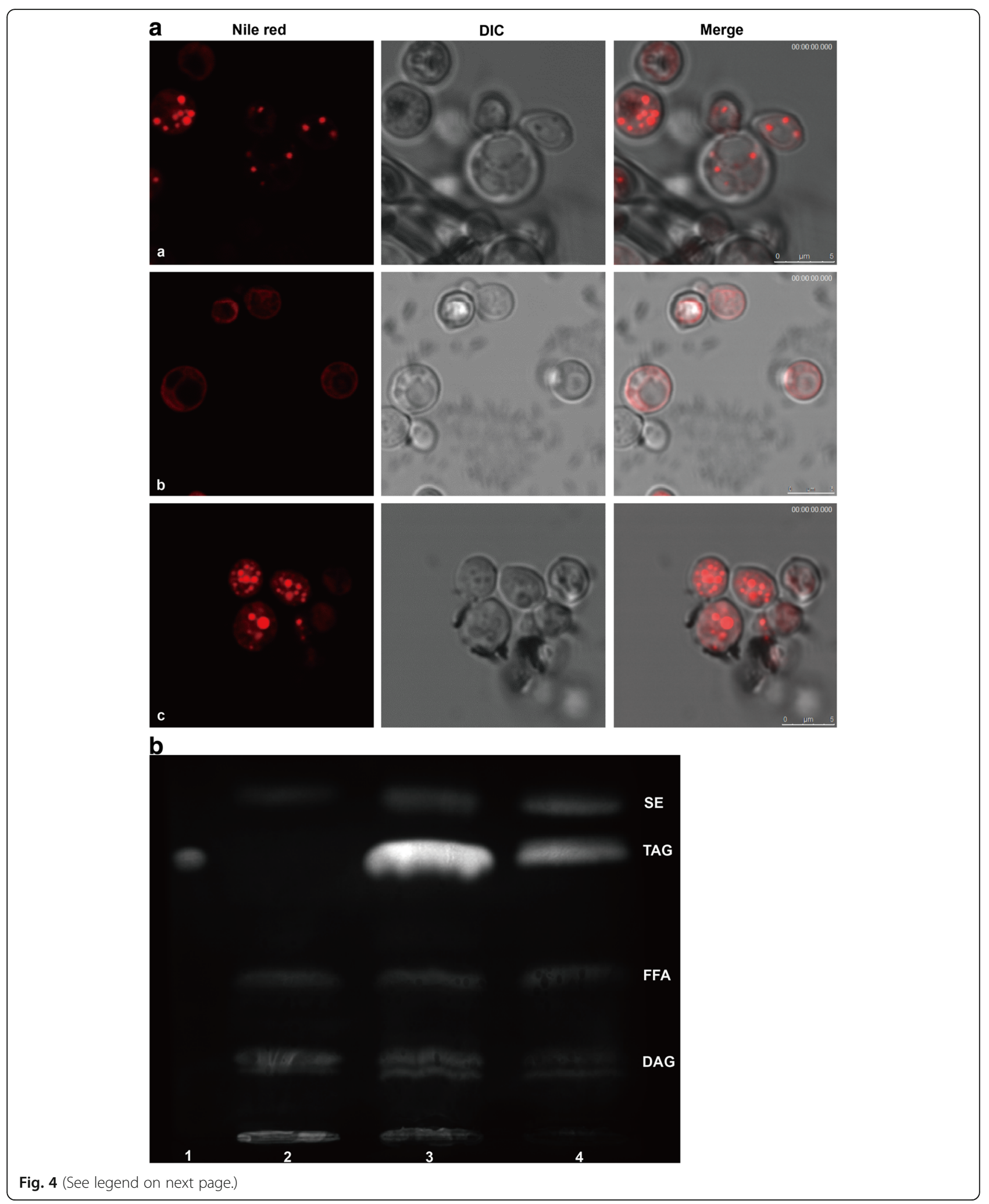


(See figure on previous page.)

Fig. 4 Nile Red staining and TLC analysis of transformed yeast strain H1246. (A) The formation of lipid bodies is restored upon the expression of CeDGAT1 in the yeast strain H1246. Nile Red staining (left) and interference contrast images (right) of yeast. The wild-type INVSc1 harbouring the empty vector was used as a positive control (a). The mutant strain $\mathrm{H} 1246$ harbouring the empty vector was analysed as a negative control (b). The TAG-deficient quadruple mutant strain H1246 expressing CeDGAT1 (c). Bar $=2.5 \mu \mathrm{m}$. (B) TLC analysis of lipid extracts from yeast expressing CeDGAT1. (1) TAG marker; (2) The mutant strain H1246 harbouring the empty vector analysed as a negative control; (3) Mutant strain H1246 expressing CeDGAT1; and (4) The wild-type strain INVSC1 harbouring the empty plasmid used as a positive control. SE, steryl ester; TAG,

Triacylglycerol; FFA, free fatty acid; DAG, diacylglycerol

characterization in yeast showed that CeDGAT1 can increase the TAG content more than can AtDGAT1, GmDGAT1 and BoDGAT1, resulting in a significant increase in the total lipid content of yeast of $142 \%$. Further investigations of the relationships between the CeDGAT1 activity and structure are needed. Its higher activity provides a scientific and economic basis for the use of $C$. ellipsoidea as an oil-producing alga to produce more oil in a short time.

In higher plants, the expression of DGAT generally correlates with oil deposition in developing seeds [21]. For soybeans, a stronger expression of DGAT1 was found in developing seeds than in other tissues [13]. However, DGAT1 transcripts were also detected in other plant tissues, e.g., AtDGAT was expressed in a wide range of tissues but most strongly in developing embryos and flower petals [18]. DGAT1 is also highly expressed during pollen development, presumably contributing to TAG accumulation in the pollen grain [48]. These findings suggested that these DGAT enzymes may be related to other physiological processes in addition to seed oil

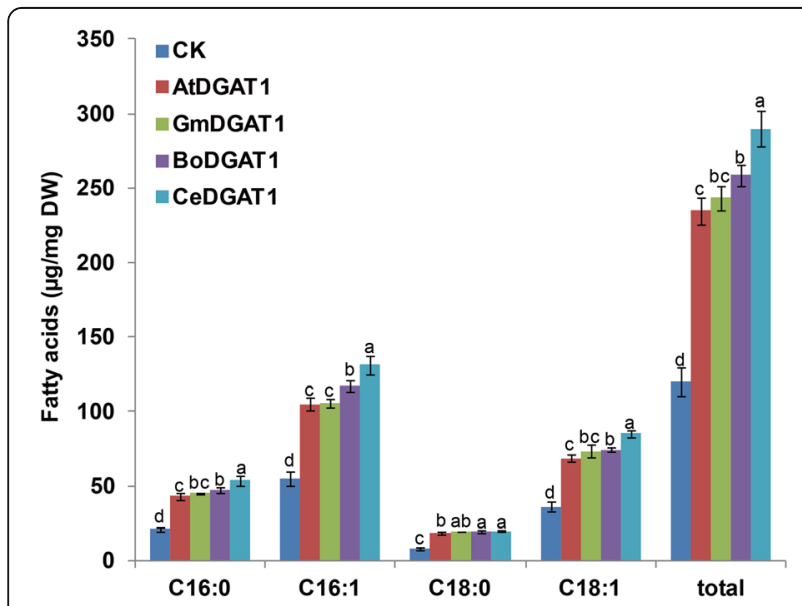

Fig. 5 Fatty acids analysis of the wild-type yeast INVSC1 expressing DGAT1s. CK, the wild-type yeast INVSC1 transformed with pYES2.0; AtDGAT1, GmDGAT1, BoDGAT1, and CeDGAT1 are transgenic yeast INVSC1 expressing AtDGAT1, GMDGAT1, BODGAT1 and CeDGAT1, respectively; C16:0, palmitic acid; C16:1, palmitoleic acid; C18:0 stearic acid; C18:1, oleic acid; and total, the sum of the C16:0, C16:1, C18:0 and C18:1 contents. The bars are the standard deviations (SDs) of three technical repeats. For the same fatty acid component, the numbers with different letters are statistically significant $(P<0.05)$ synthesis [15]. For unicellular eukaryotic green algae, all physiological processes take place within a cell; thus, the expression of DGAT1 can directly reflect the dynamics of TAG accumulation.

The effect of nutrition pattern alteration on algal cell growth, lipid accumulation, and cellular component

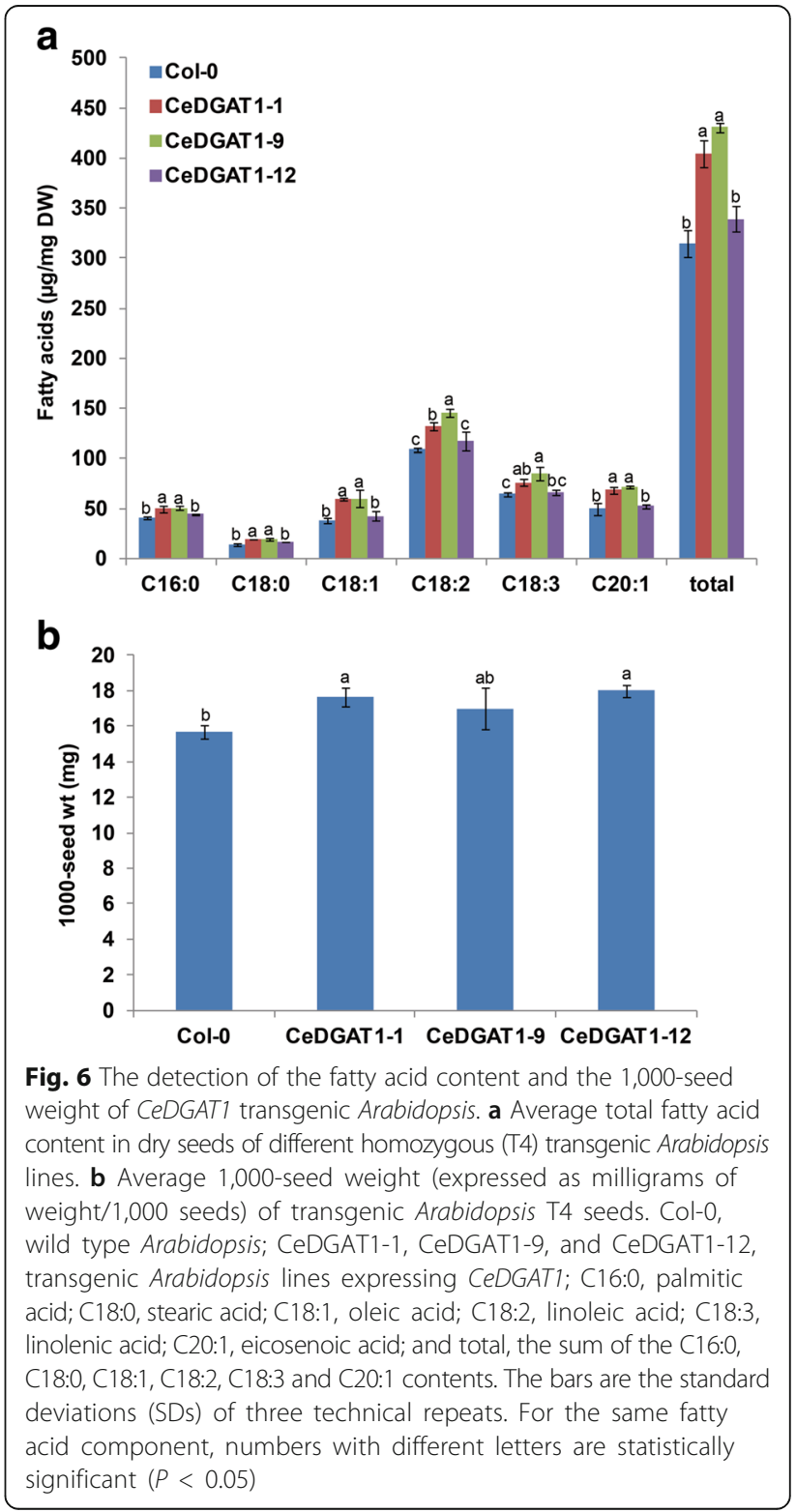




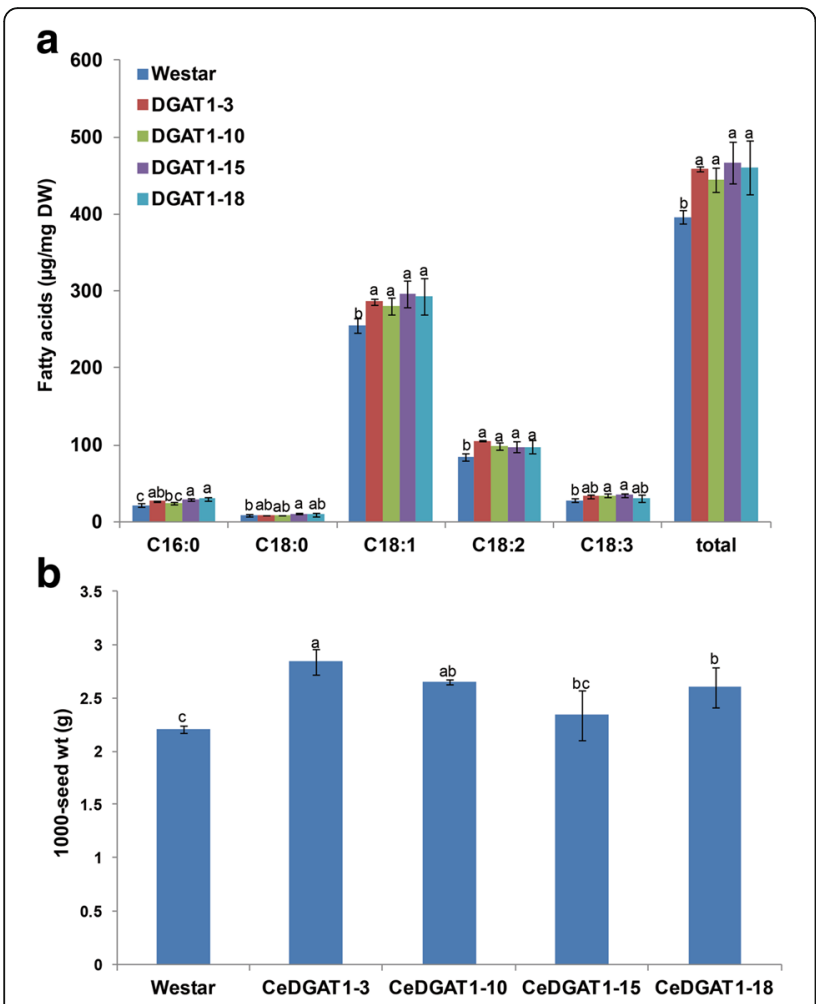

Fig. 7 The detection of the fatty acid content and the 1,000-seed weight of CeDGAT1 transgenic B. napus. a Average total fatty acid content in dry seeds of different (T3) transgenic $B$. napus lines. $\mathbf{b}$ Average 1,000-seed weight (expressed as grams of weight/1,000 seeds) of transgenic $B$. napus $T 3$ seeds. Westar, wild type B. napus var. Westar; CeDGAT1-3, CeDGAT1-10, CeDGAT1-15, and CeDGAT1-18, transgenic $B$. napus lines expressing CeDGAT1; C16:0, palmitic acid; C18:0, stearic acid; C18:1, oleic acid; C18:2, linoleic acid; C18:3, linolenic acid; and total, the sum of the C16:0, C18:0, C18:1, C18:2 and C18:3 contents. The bars are the standard deviations (SDs) of three technical repeats. For the same fatty acid component, numbers with different letters are statistically significant $(P<0.05)$

changes has been analysed in several studies [49-54]. Upon nitrogen starvation, both starches and lipids increased greatly within C. zofingiensis [51] and Nannochloropsis oceanica cells [54], and N-deficiency plus P-repletion was a promising lipid trigger to motivate lipid accumulation in C. protothecoides cells [50]. In C. reinhardtii, three genes encoding acyltransferases, DGAT1, DGTT1, and $P D A T 1$, were induced by nitrogen starvation and are likely to play a role in TAG accumulation based on their patterns of expression [39]. At the transcript level in $N$. oceanica, enhanced TAG synthesis under N-depleted conditions primarily involved the upregulation of seven putative DGAT genes and the downregulation of six other DGAT genes [55]. However, the expression patterns of lipid biosynthesisrelated genes, including DGAT1 in Chlorella, have not been extensively studied in these processes. Our results revealed that the upregulation of CeDGAT1 was closely related to the significant increase in the TFA and TAG contents under nitrogen deprivation, suggesting that $C e D G A T 1$ plays an important role in TAG accumulation. Our findings contribute to the understanding of the microalgal response to element deprivation and the mechanism of lipid synthesis and accumulation in Chlorella, but much remains to be elucidated regarding the precise contribution of $\mathrm{N}$ starvation to microalgal metabolism.

Several previous studies have reported that the genetic manipulation of DGAT can lead to increased oil biosynthesis in Arabidopsis and B. napus. For instance, the seed-specific over-expression of $A$. thaliana DGAT1 in wild-type Arabidopsis can increase the seed oil content by $11-28 \%$ and the seed weight by $2.5-32.3 \%$ [43]. Similarly, the seed-specific expression of TmDGAT1 in wildtype Arabidopsis resulted in a $10-33 \%$ net increase in the seed oil content and a 15\% increase in the 1,000-seed weight in transgenic Arabidopsis. Furthermore, the seedspecific expression of TmDGAT1 in high-erucic acid $B$. napus led to a net increase of $11-15 \%$ in the seed oil content of transgenic plants [6]. In addition, the overexpression of AtDGAT1 and BnDGAT1 in canola under the control of the napin promoter led to an increase of $2.5-7 \%$ in the oil content [56]. The over-expression of JcDGAT1 in Arabidopsis under both CaMV35S promoter and a seed specific promoter resulted in a $30-41 \%$ increase in the seed oil content [25]. Our studies showed that the expression of CeDGAT1 in Arabidopsis and B. napus under the NOS promoter does indeed increase oil biosynthesis in transgenic seeds by approximately $8-37 \%$ and $12-18 \%$ over that of the control. In addition, neither

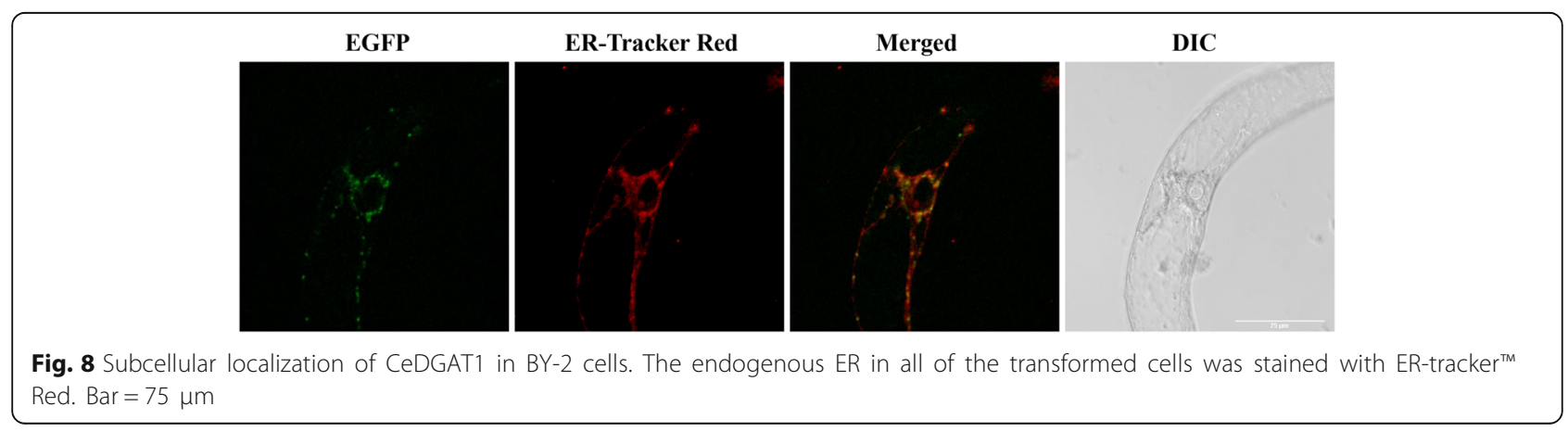


the Arabidopsis nor the B. napus CeDGAT1 transformants showed significant changes in fatty acid composition. In some studies, however, there were alterations in the oil composition through DGAT expression. The over-expression of JcDGAT1 in Arabidopsis resulted in a significant decrease in oleic acid (C18:1) and an increase in linolenic acid (C18:3) [25], and the transgenic expression of Sesamum indicum DGAT1 in Arabidopsis led to an increase in eicosenoic acid (C20:1) and a reduction in oleic acid (C18:1) in seed oil [26]. More importantly, the expression of CeDGAT1 in Arabidopsis and B. napus under the NOS promoter also led to a significant increase in the average 1,000-seed weight in CeDGAT1 transgenic lines, by $9-15 \%$ and $6-29 \%$ in Arabidopsis and $B$. napus, respectively, and thus there was no decrease in the 1,000-seed weight caused by the oil content increase. Considering the constitutive expression of CeDGAT1 under the NOS promoter, larger increments in seed oil biosynthesis and seed weight can probably be expected when using a seed specific promoter. Furthermore, there was a difference between CeDGAT1 transgenic Arabidopsis and B. napus, with respect to effects on seed oil biosynthesis and seed weight. The seed oil content increased more in transgenic Arabidopsis than in $B$. napus, but the average seed weight increase was greater in B. napus. Interestingly, the increase in the oil content on a per-1,000-seed basis was similar between transgenic Arabidopsis and B. napus, at approximately $25-50 \%$. To date, there has been no report that the over-expression of DGAT1 can significantly increase the seed weight in the oil plant $B$. napus, although this effect has been reported in Arabidopsis. In addition, transgenic plants showed no other phenotypic differences. Therefore, CeDGAT1 should have great potential for increasing the net oil production of the oil plant oilseed rape.

\section{Conclusion}

We cloned a novel DGAT1 gene (CeDGAT1) from $C$. ellipsoidea. CeDGAT1 is novel protein, sharing a low identity $(\leq 40 \%)$ with DGAT1s from higher plants. The expression of CeDGAT1 is highly related to rapid lipid accumulation in C. ellipsoidea under nitrogen deprivation. In yeast, the expression of CeDGAT1 can significantly increase the lipid content and shows greater ability for improving the lipid synthesis than DGAT1s from some higher plants, including that from soybean. Moreover, the expression of CeDGAT1 in Arabidopsis and oilseed rape can lead to a net increase in the 1,000 -seed lipid content of transgenic plants of 25$50 \%$. These findings should be helpful for understanding the function of DGAT from microalgae and the mechanism of lipid synthesis and accumulation and may also provide technology for enhancing lipid production in microalgae and oil plants.

\section{Methods}

\section{Strains and growth conditions}

Chlorella ellipsoidea was initially cultured mixotrophically in $1 \mathrm{~L}$ flasks containing $500 \mathrm{~mL}$ of sterilized Endo medium [57] and incubated at $25^{\circ} \mathrm{C}$ under illumination $\left(100 \mu \mathrm{mol}\right.$ photons $\left./ \mathrm{m}^{2} / \mathrm{s}\right)$ for one week with shaking at $160 \mathrm{r} / \mathrm{min}$. These pre-cultured cells were transferred to Erlenmeyer flasks $(3 \mathrm{~L})$, each containing $1 \mathrm{~L}$ of fresh medium to a final volume of $1.5 \mathrm{~L}$, and incubated for another 4 days. These pre-cultured cells, after centrifugation and washing with sterilized water, were sampled as the starting point $(0 \mathrm{~h})$. Then, the collected cells were resuspended at a density of approximately $9.5 \mathrm{~g} / \mathrm{L}$ in a 20 L BioFlo 415 fermentor (New Brunswick Scientific, USA) containing $14 \mathrm{~L}$ of modified Endo medium, in which urea was used to replace $\mathrm{KNO}_{3}$ in the original Endo medium. The media containing urea at $0.4 \mathrm{~g} / \mathrm{L}$ and $1.6 \mathrm{~g} / \mathrm{L}$ were named $\mathrm{N}$-depleted $(1 / 4 \mathrm{~N})$ and $\mathrm{N}$ repletion media, respectively. The culture conditions were maintained at $25{ }^{\circ} \mathrm{C}$, and a thermocirculator was used to maintain a constant temperature in the fermentor by circulating water through the jacket. The fermentor was aerated with filtered ambient air at a flow rate of $0.5 \mathrm{vvm}$, and the $\mathrm{pH}$ was maintained at 6.8 using $1 \mathrm{M}$ $\mathrm{KOH}$. The cultures were sampled $24,36,60,84$ and $108 \mathrm{~h}$ after they were initiated using $\mathrm{N}$-depleted $(1 / 4 \mathrm{~N})$ or N-repletion medium. The cells were harvested by centrifugation $\left(5,000 \mathrm{~g}\right.$ at $4{ }^{\circ} \mathrm{C}$ for $\left.5 \mathrm{~min}\right)$. Aliquots for RNA analysis and gene cloning were frozen in liquid nitrogen and stored at $-80{ }^{\circ} \mathrm{C}$ if not immediately used, while those for lipid analysis were washed with water and freeze-dried.

The wild-type yeast strain INVSc1 (Invitrogen, UK), the H1246 mutant strain (Matayor245c::KanMX4 lro1::TRP1 are 1::HIS3 are2::LEL2 ADE2 ura3) [47], Arabidopsis thaliana (ecotype Columbia) and Brassica napus (Westar) were used to determine the function of CeDGAT1 by heterologous expression.

\section{Cloning of a CDNA encoding DGAT1 from C. ellipsoidea}

Total RNA was isolated from algae cells of the exponential growth phase of C. ellipsoidea using the EasySpin RNA Extraction Kit (Aidlab Biotech, Beijing, China), and cDNA was prepared from $5 \mu \mathrm{g}$ of total RNA-template with the ReverTra Ace qPCR RT Kit (Toyobo, Osaka, Japan). The coding sequence of CeDGAT1 was amplified using the gene-specific primers P1 and P2 (Additional file 6: Table S2) based on the expressed sequence tag (EST) data of C. ellipsoidea. The $25 \mu \mathrm{L}$ final reaction volume used for PCR contained $2.5 \mu \mathrm{L}$ of $10 \times$ PCR buffer with $\mathrm{MgCl}_{2}, 1 \mu \mathrm{L}$ of each primer $(10 \mu \mathrm{M}), 2.0 \mu \mathrm{L}$ of $2.5 \mathrm{mM}$ dNTPs, $1 \mu \mathrm{L}$ of cDNA sample, $0.5 \mu \mathrm{L}$ of EasyPfu DNA polymerase (TransGen Biotech, Beijing, China), and $17 \mu \mathrm{L}$ of double-distilled water. The reaction 
conditions for PCR were as follows: denatured at $95{ }^{\circ} \mathrm{C}$ for $10 \mathrm{~min}$, followed by 30 cycles of $94{ }^{\circ} \mathrm{C}$ for $30 \mathrm{~s}, 55^{\circ} \mathrm{C}$ for $30 \mathrm{~s}$, and $72{ }^{\circ} \mathrm{C}$ for $2 \mathrm{~min}$; and a final extension step of $72{ }^{\circ} \mathrm{C}$ for $10 \mathrm{~min}$. The amplified cDNA was cloned into the $p E A S Y$-Blunt vector (TransGen Biotech, Beijing, China), and the corresponding clones were verified by PCR and DNA sequencing.

\section{Yeast expression vector construction and transformation}

The full-length CeDGAT1 ORF was amplified using primers P3 and P4 (Additional file 6: Table S2) and subcloned between the Hind III and EcoR I sites of the pYES2.0 yeast expression vector. The Saccharomyces cerevisiae strains (the wild-type strain INVSc1 and the mutant strain H1246) were transformed using the LiAc method [58]. We also separately transferred another three DGAT1 genes from higher plants, including the oil crop Glycine $\max$ (accession no. AY496439.1), Arabidopsis thaliana (accession no. NM_127503.2) and Brassica oleracea (unpublished data from our laboratory) into the wild-type yeast (INVSc1). The primers that were used for DGAT1 gene cloning are shown in Additional file 6: Table S2. Transformants were selected on synthetic complete medium lacking uracil (SC-ura). For heterologous expression studies, the yeast strains were transferred into liquid SC-ura medium containing $2 \%$ $(\mathrm{w} / \mathrm{v})$ glucose at $30{ }^{\circ} \mathrm{C}$ overnight and then induced by adding $2 \%(\mathrm{w} / \mathrm{v})$ galactose and $1 \%(\mathrm{w} / \mathrm{v})$ tergitol NP-40 (Sigma, Taufkirchen, Germany) for an additional $72 \mathrm{~h}$ at $20{ }^{\circ} \mathrm{C}$. The expression of DGAT1s in transgenic yeast was verified at the transcript level by RT-PCR (for the RT-PCR primers see Additional file 6: Table S2).

\section{Plant vector construction and transformation}

The complete CeDGAT1 was cloned into the plant expression vector pCAMBIA2301 under the control of the nopaline synthase (NOS) promoter and nos terminator, yielding pCAMBIA2301-CeDGAT1 (Additional file 7: Figure S5). The final binary vector was verified and then transferred into Agrobacterium tumefaciens strain GV3101 by the freeze-thaw method [59]. Arabidopsis plants were transformed by vacuum infiltration [60]. Brassica napus var. Westar was transformed using hypocotyl explants and the modified method of DeBlock et al. [61]. T1 generation seeds were selected on kanamycin $(50 \mathrm{mg} / \mathrm{L})$, and then the selected transformed plants were transferred to soil. T3 transgenic B. napus lines and homozygous T4 transgenic Arabidopsis lines were used for seed and oil analyses. Genomic DNA was isolated from B. napus var. Westar leaf material. The stable integration of the NOS: CeDGAT1: nos cassette into the genome of transgenic $B$. napus was checked by PCR amplification using the specific primers P21 and P22 (Additional file 6: Table S2). GUS histochemical staining of the leaves from the transgenic lines was also conducted as described by Jefferson et al. [62]. In the meantime, the expression of CeDGAT1 in Arabidopsis and B. napus was detected by RT-PCR using the CeDGAT1specific primers P11 and P12 (Additional file 6: Table S2). The Arabidopsis housekeeping gene actin (primers P23 and P24) and the B. napus housekeeping gene GAPDH (primers P25 and P26) were used as internal controls.

\section{Nile Red staining and microscopy}

The Nile Red staining was used to visualize the intracellular lipid bodies as an indicators of TAG formation [63]. For yeast cell staining, a $500 \mu \mathrm{L}$ suspension of yeast cells in the culture medium was stained with $5 \mu \mathrm{L}$ of Nile Red ( $1 \mathrm{mg} / \mathrm{mL}$ in acetone stock), incubated in the dark for $5 \mathrm{~min}$, and immediately used for microscopic analysis.

\section{Lipid analysis by TLC and GC-MS}

For the analysis of lipids from yeast and C. ellipsoidea, the cells were harvested by centrifugation, and the resulting cell pellets were ground to a fine powder under liquid nitrogen and subsequently treated with isopropanol at $80{ }^{\circ} \mathrm{C}$ for $10-15$ min to stop the lipolytic activity. Isopropanol was evaporated under nitrogen gas before lipid extraction. The total lipids were extracted according to a modified version of the Bligh and Dyer method [64], and TAG was separated from the total lipids by thin-layer chromatography (TLC) on Silica Gel 60 plates (Merck, Darmstadt, Germany). The solvents that were used were hexane/diethyl ether/glacial acetic acid (70:30:1, $\mathrm{v} / \mathrm{v})$. The lipids were visualized by spraying Primuline (Sigma, $10 \mathrm{mg} / 100 \mathrm{~mL}$ acetone: water $(60: 40 \mathrm{v} / \mathrm{v})$ ) and exposing the plate to UV. Triolein (Sigma) was used as the standard. TAGs were recovered from the TLC plates and then trans-esterified with $5 \% \mathrm{H}_{2} \mathrm{SO}_{4}$ in methanol at $85^{\circ} \mathrm{C}$ for $1 \mathrm{~h}$. The fatty acid methyl esters (FAMEs) were extracted with hexane and analysed by GC-MS following the methods described in the following section.

\section{Fatty acid analysis}

Cellular fatty acids were extracted by incubating $10 \mathrm{mg}$ of dried seeds of control and transformed plants or $50 \mathrm{mg}$ of yeast powder and freeze-dried algae powder in $3 \mathrm{~mL}$ of $7.5 \%(\mathrm{w} / \mathrm{v}) \mathrm{KOH}$ in methanol for saponification at $70{ }^{\circ} \mathrm{C}$ for $4 \mathrm{~h}$. After the $\mathrm{pH}$ was adjusted to 2.0 with $\mathrm{HCl}$, the fatty acid were subjected to methylesterification with $2 \mathrm{~mL}$ of $14 \%(\mathrm{w} / \mathrm{v})$ boron trifluoride in methanol at $70{ }^{\circ} \mathrm{C}$ for $1.5 \mathrm{~h}$. A phase separation was produced by adding $1 \mathrm{~mL}$ of $0.9 \%(\mathrm{w} / \mathrm{v}) \mathrm{NaCl}$ and $4 \mathrm{~mL}$ of hexane. The upper phase was dried under a nitrogen gas flow and resuspended in $0.3 \mathrm{~mL}$ of acetic ether prior to GC analysis. An analysis of fatty acid methyl esters (FAME) was performed by GC-MS (gas chromatography-mass spectrometry, TurboMass, PerkinElmer, USA) equipped 
with a capillary column (BPX-70, $30 \mathrm{~m} \times 0.25 \mathrm{~mm} \times$ $0.25 \mu \mathrm{m})$. Hydrogen was used as the carrier gas at a flow rate of $1.0 \mathrm{~mL} / \mathrm{min}$. The injector and detector temperatures were held at $250{ }^{\circ} \mathrm{C}$. The column oven was temperatureprogrammed from 100 to $190{ }^{\circ} \mathrm{C}$ at $15{ }^{\circ} \mathrm{C} / \mathrm{min}$, where the temperature was held for $1 \mathrm{~min}$ increased to $220^{\circ} \mathrm{C}$ at $10{ }^{\circ} \mathrm{C} / \mathrm{min}$, and then held for $4 \mathrm{~min}$. The total FA content was quantified using heptadecanoic acid (C17:0) (Sigma) as an internal standard added to samples prior to extraction.

\section{Dry weight determination}

For dry weight determination, the algal cells were collected by filtering the culture through pre-weighed Whatman GF/C filter paper $(1.2 \mu \mathrm{m}$ pore size). Then, the filter paper was dried at $80{ }^{\circ} \mathrm{C}$ in an oven until the weight was constant.

The C. ellipsoidea biomass concentration $(\mathrm{w} / \mathrm{v})$ was equivalent to a specific value of the cell dry weight (DW) that was determined by $\mathrm{OD}_{540}$ according to the following empirical formula:

$$
\mathrm{DW}(\mathrm{g} / \mathrm{L})=\left(\mathrm{OD}_{540}+0.0097\right) / 0.4165
$$

\section{Quantitative real-time PCR detection of CeDGAT1 expression in C. ellipsoidea}

The total RNA was isolated from the cells of $C$. ellipsoidea at six growing points $(0 \mathrm{~h}, 24 \mathrm{~h}, 36 \mathrm{~h}, 60 \mathrm{~h}, 84 \mathrm{~h}$, and $108 \mathrm{~h}$ ) during the time course of nitrogen depletion or repletion. All of the real-time reactions were performed on a LightCycler 480 Real-Time PCR System (Roche Applied Science, Mannheim, Germany) using the LightCycler 480 SYBR Green I Master Mix Kit (Roche Applied Science) according to the manufacturer's instructions: $95{ }^{\circ} \mathrm{C}$ for $30 \mathrm{~s}$ and then 40 cycles of $95^{\circ} \mathrm{C}$ for $10 \mathrm{~s}$, followed by $55{ }^{\circ} \mathrm{C}$ for $10 \mathrm{~s}$, and $72{ }^{\circ} \mathrm{C}$ for $20 \mathrm{~s}$. All of the qRT-PCR experiments were performed in triplicate. The primers that were used for the qRT-PCR of CeDGAT1 are P27 and P28 (Additional file 6: Table S2). To normalize the transcript levels in each sample, $18 \mathrm{~S}$ rRNA was used as the internal standard (primers P29 and P30). The relative expression was computed following the formula (Cta-Ctb), where $\mathrm{Cta}$ and $\mathrm{Ctb}$ are the average $\mathrm{Ct}$ values of the reference and target genes, respectively.

\section{Alignment and molecular phylogenetic analysis}

Multiple alignments were performed using MAFFT v6.847b [65] with the L-INS-i algorithm. A phylogenetic tree was reconstructed with FastTree using the approximate maximum-likelihood method [66]. For testing the robustness of the tree, 1000 bootstrap replicates were carried out. The transmembrane regions in the CeDGAT1 protein were predicted with the TMpred program (http://www.ch.embnet.org/software/TMPRED_ form.html). Three-dimensional structures of the DGAT1 proteins (CeDGAT1, AtDGAT1, GmDGAT1, and BoDGAT1) were predicted by I-TASSER (http://zhanglab.ccmb. med.umich.edu/I-TASSER/).

Subcellular localization of CeDGAT1 in tobacco BY-2 Cells To determine its subcellular location, CeDGAT1 without the stop codon was amplified from cDNA, cloned into the GATEWAY donor vector pGWC according to the method described by Chen et al. [67] and sequenced. The CeDGAT1 was then introduced in the destination vector pGWB5 with infusion with EGFP under the CaMV 35S promoter by LR reaction following the manufacturer's instructions (Invitrogen). The final construct CeDGAT1::EGFP was transferred into Agrobacterium tumefaciens strain GV3101. The Agrobacterium-mediated transformation of tobacco BY2 cells was performed according to An [68] and Genschik et al. [69]. Briefly, BY2 cells were incubated for 3 days at $27{ }^{\circ} \mathrm{C}$ in the dark (without shaking) with Agrobacterium GV3101 containing CeDGAT1::EGFP. Subsequently, cells were plated on medium containing two antibiotics: timentin $(500 \mathrm{mg} / \mathrm{L})$ to kill off Agrobacterium and kanamycin $(100 \mathrm{mg} / \mathrm{L})$ to select for transformed cells. Transformed cells appeared after one month as a callus on plates and were then transferred to fresh plates once a month. A suspension culture was obtained by the addition of small transformed callus clumps to liquid culture medium containing kanamycin. The transformed BY-2 suspension cells were stained with ER-tracker ${ }^{\mathrm{Tw}}$ Red following the manufacturer's instructions (Invitrogen) and then observed and photographed using a Leica TCS SP5 confocal laser scanning microscope (Leica Microsystems, Germany).

\section{Statistical analysis}

All of the experimental data were statistically compared using a one-way analysis of variance (ANOVA) with the software Statistical Product and Service Solutions (SPSS) v19.0, followed by a post-hoc test to determine the significant difference among the treatment means.

\section{Additional files}

Additional file 1: Table S1. Putative functional motifs in CeDGAT1 and AtDGAT1. (DOCX $17 \mathrm{~kb}$ )

Additional file 2: Figure S1. RT-PCR detection of DGAT1 genes in transgenic yeast (INVSC1). The yeast actin was used as an internal control. 1, The yeast transformed with pYES2.0; 2-5, the yeast expressing AtDGAT1, GmDGAT1, BnDGAT1 and CeDGAT1, respectively. (DOCX $55 \mathrm{~kb}$ )

Additional file 3: Figure S2. A schematic map of the PCAMBIA2301NOS-CeDGAT1-nos plasmid. The pCAMBIA2301-NOS-CeDGAT1-nos vector contained an expression box of the CeDGAT1 gene from C. ellipsoidea under the control of the NOS promoter and nos terminator; an expression box of the GUS gene controlled by the CaMV35S promoter and nos 
terminator; and an expression box of the kanamycin resistance gene, which conferred resistance to kanamycin. (DOCX $243 \mathrm{~kb}$ )

Additional file 4: Figure S3. RT-PCR detection of CeDGAT1 in transgenic Arabidopsis (A) and $B$. napus (B) lines. Arabidopsis actin and B. napus GAPDH were used as internal controls. Col-0, wild-type Arabidopsis; 1, 9, and 12, transgenic Arabidopsis lines expressing NOS:CeDGAT1; Westar, wild-type $B$. napus var. Westar; $3,10,15$, and 18 , transgenic $B$. napus lines expressing NOS:CeDGAT1. (DOCX 93 kb)

Additional file 5: Figure S4. PCR detection of the CeDGAT1 gene in transgenic $B$. napus lines. M, DNA molecular weight marker; 1, blank control; 2, wild-type B. napus var. Westar; 3-14, lines expressing NOS:CeDGAT1; 15, pCAMBIA2301-NOS-CeDGAT1-nos plasmid (positive control). (DOCX $112 \mathrm{~kb}$ )

Additional file 6: Table S2. List of primers. Sequences in lower-case letters indicate enzyme restriction sites. (DOCX $14 \mathrm{~kb}$ )

Additional file 7: Figure S5. Comparison of the predicted structures of CeDGAT1, AtDGAT1, GmDGAT1 and BoDGAT1. (DOCX $416 \mathrm{~kb}$ )

\section{Acknowledgements}

We thank Dr. Peng Jiang (the Key Laboratory of Experimental Marine Biology, Institute of Oceanology, Chinese Academy of Sciences) for providing the yeast mutant strain H1246 (Matayor245c::KanMX4 Iro1::TRP1 are1::HIS3 are2::LEU2 ADE2 ura3). We also thank Dr. Liying Song (Institute of Genetics and Developmental Biology, Chinese Academy of Sciences) for her technical help.

\section{Funding}

This work was supported by a project (2016ZX08009-003) from the Agriculture Ministry of China and projects (31570365 and 21306222) from the National Natural Science Foundation of China.

\section{Availability of data and materials}

The datasets supporting the results of this article are included within the article and its additional files. We deposited the phylogenetic tree, sequence data and alignments used to produce the results displayed in Fig. 1 in TreeBASE (http://purl.org/phylo/treebase/phylows/study/TB2:S19951), where it will be made freely available

\section{Authors' contributions}

XG carried out the experiments, analysed the data and drafted the manuscript. CF and YC participated in the experimental design and performed phylogenetic analyses of DGAT. WY performed phylogenetic analyses of DGAT and detected the expression of DGAT1s in yeast. JW and RW gave some good advice for writing the manuscript. ZH conceived the study, participated in its design and revised the manuscript. All of the authors read and approved the final manuscript.

\section{Competing interests}

The authors declare that they have no competing interests.

\section{Consent for publication}

Not applicable.

\section{Ethics approval and consent to participate}

Not applicable.

\section{Author details}

'Institute of Genetics and Developmental Biology, Chinese Academy of Sciences, Beijing 100101, China. ${ }^{2}$ University of Chinese Academy of Sciences, Beijing 100049, China. ${ }^{3}$ Institute of Economical Crops, Yunnan Agricultural Academy, Kunming 65023, China. ${ }^{4}$ United States Department of Agriculture, Agricultural Research Service, Forage and Range Research Laboratory, Utah State University, Logan, UT 84322-6300, USA. ${ }^{5}$ Present address: Institute of Genetics and Developmental Biology, Chinese Academy of Sciences, Datun Road, Chaoyang District, Beijing 100101, China.
Received: 4 September 2016 Accepted: 2 February 2017

Published online: 21 February 2017

\section{References}

1. Durrett TP, Benning C, Ohlrogge J. Plant triacylglycerols as feedstocks for the production of biofuels. Plant J. 2008:54:593-607.

2. Dyer JM, Stymne S, Green AG, Carlsson AS. High-value oils from plants. Plant J. 2008;54:640-55

3. Huang AHC. Oleosins and oil bodies in seeds and other organs. Plant Physiol. 1996;110:1055-61.

4. Chapman KD, Dyer JM, Mullen RT. Biogenesis and functions of lipid droplets in plants: thematic review series: lipid droplet synthesis and metabolism: from yeast to man. J Lipid Res. 2012:53:215-26.

5. Nicole K, Simoni RD, Hill RL. Otto Fritz Meyerhof and the elucidation of the glycolytic pathway. J Biol Chem. 2005;280:e3.

6. Xu J, Francis T, Mietkiewska E, Giblin EM, Barton DL, Zhang Y, et al. Cloning and characterization of an acyl-CoA-dependent diacylglycerol acyltransferase 1 (DGAT1) gene from Tropaeolum majus, and a study of the functional motifs of the DGAT protein using site-directed mutagenesis to modify enzyme activity and oil content. Plant Biotechnol J. 2008:6:799-818.

7. Ichihara Kl, Takahashi T, Fujii S. Diacylglycerol acyltransferase in maturing safflower seeds: its influences on the fatty acid composition of triacylglycerol and on the rate of triacylglycerol synthesis. Biochim Biophys Acta. 1988; 958:125-9.

8. Settlage SB, Kwanyuen P, Wilson RF. Relation between diacylglycerol acyltransferase activity and oil concentration in soybean. J Am Oil Chem Soc. 1998;75:775-81.

9. Turchetto-Zolet AC, Maraschin FS, de Morais GL, Cagliari A, Andrade CM, Margis-Pinheiro $\mathrm{M}$, et al. Evolutionary view of acyl-CoA diacylglycerol acyltransferase (DGAT), a key enzyme in neutral lipid biosynthesis. BMC Evol Biol. 2011;11:263.

10. Shockey JM, Gidda SK, Chapital DC, Kuan JC, Dhanoa PK, Bland JM, et al. Tung tree DGAT1 and DGAT2 have nonredundant functions in triacylglycerol biosynthesis and are localized to different subdomains of the endoplasmic reticulum. Plant Cell. 2006:18:2294-313.

11. Kroon JT, Wei W, Simon WJ, Slabas AR. Identification and functional expression of a type 2 acyl-CoA:diacylglycerol acyltransferase (DGAT2) in developing castor bean seeds which has high homology to the major triglyceride biosynthetic enzyme of fungi and animals. Phytochemistry. 2006;67:2541-9.

12. Cahoon EB, Shockey JM, Dietrich CR, Gidda SK, Mullen RT, Dyer JM. Engineering oilseeds for sustainable production of industrial and nutritional feedstocks: solving bottlenecks in fatty acid flux. Curr Opin Plant Biol. 2007;10:236-44.

13. Li R, Yu K, Hatanaka T, Hildebrand DF. Vernonia DGATs increase accumulation of epoxy fatty acids in oil. Plant Biotechnol J. 2010;8:184-95.

14. Yen CLE, Stone SJ, Koliwad S, Harris C, Farese RV. DGAT enzymes and triacylglycerol biosynthesis. J Lipid Res. 2008;49:2283-301.

15. Liu Q, Siloto RM, Lehner R, Stone SJ, Weselake RJ. Acyl-CoA:diacylglycerol acyltransferase: molecular biology, biochemistry and biotechnology. Prog Lipid Res. 2012;51:350-77.

16. Guiheneuf F, Leu S, Zarka A, Khozin-Goldberg I, Khalilov I, Boussiba S. Cloning and molecular characterization of a novel acyl-CoA:diacylglycerol acyltransferase 1-like gene (PtDGAT1) from the diatom Phaeodactylum tricornutum. FEBS J. 2011:278:3651-66.

17. Cases S, Novak S, Zheng YW, Myers HM, Lear SR, Sande E, et al. ACAT-2, a second mammalian acyl-CoA : cholesterol acyltransferase - its cloning expression, and characterization. J Biol Chem. 1998;273:26755-64.

18. Hobbs DH, Lu CF, Hills MJ. Cloning of a cDNA encoding diacylglycerol acyltransferase from Arabidopsis thaliana and its functional expression. FEBS Lett. 1999:452:145-9.

19. Bouvier-Nave P, Benveniste $P$, Oelkers $P$, Sturley SL, Schaller H. Expression in yeast and tobacco of plant cDNAs encoding acyl CoA : diacylglycerol acyltransferase. Eur J Biochem. 2000;267:85-96.

20. Nykiforuk CL, Furukawa-Stoffer TL, Huff PW, Sarna M, Laroche A, Moloney $\mathrm{MM}$, et al. Characterization of CDNAs encoding diacylglycerol acyltransferase from cultures of Brassica napus and sucrose-mediated induction of enzyme biosynthesis. Biochim Biophys Acta. 2002;1580:95-109.

21. He XH, Turner C, Chen GQ, Lin JT, McKeon TA. Cloning and characterization of a cDNA encoding diacylglycerol acyltransferase from castor bean. Lipids. 2004;39:311-8

22. Milcamps A, Tumaney AW, Paddock T, Pan DA, Ohlrogge J, Pollard M Isolation of a gene encoding a 1,2-diacylglycerol-sn-acetyl-CoA 
acetyltransferase from developing seeds of Euonymus alatus. J Biol Chem. 2005:280:5370-7.

23. Wang HW, Zhang JS, Gai JY, Chen SY. Cloning and comparative analysis of the gene encoding diacylglycerol acyltransferase from wild type and cultivated soybean. Theor Appl Genet. 2006;112:1086-97.

24. Yu K, Li R, Hatanaka T, Hildebrand D. Cloning and functional analysis of two type 1 diacylglycerol acyltransferases from Vernonia galamensis. Phytochemistry. 2008;69:1119-27.

25. Misra A, Khan K, Niranjan A, Nath P, Sane VA. Over-expression of JcDGAT1 from Jatropha curcas increases seed oil levels and alters oil quality in transgenic Arabidopsis thaliana. Phytochemistry. 2013;96:37-45.

26. Wang Z, Huang W, Chang J, Sebastian A, Li Y, Li H, et al. Overexpression of SiDGAT1, a gene encoding acyl-CoA:diacylglycerol acyltransferase from Sesamum indicum L. increases oil content in transgenic Arabidopsis and soybean. Plant Cell Tissue Organ Cult. 2014;119:399-410.

27. Katavic V, Reed DW, Taylor DC, Giblin EM, Barton DL, Zou JT, et al. Alteration of seed fatty-acid composition by an ethyl methanesulfonate-induced mutation in Arabidopsis thaliana affecting diacylglycerol acyltransferase activity. Plant Physiol. 1995;108:399-409.

28. Andrianov V, Borisjuk N, Pogrebnyak N, Brinker A, Dixon J, Spitsin S, et al. Tobacco as a production platform for biofuel: overexpression of Arabidopsis DGAT and LEC2 genes increases accumulation and shifts the composition of lipids in green biomass. Plant Biotechnol J. 2010;8:277-87.

29. Chisti Y. Biodiesel from microalgae. Biotechnol Adv. 2007;25:294-306.

30. Hu Q, Sommerfeld M, Jarvis E, Ghirardi M, Posewitz M, Seibert M, et al. Microalgal triacylglycerols as feedstocks for biofuel production: perspectives and advances. Plant J. 2008;54:621-39.

31. Lam MK, Lee KT. Microalgae biofuels: a critical review of issues, problems and the way forward. Biotechnol Adv. 2012;30:673-90.

32. Gong Y, Zhang J, Guo X, Wan X, Liang Z, Hu CJ, et al. Identification and characterization of PtDGAT2B, an acyltransferase of the DGAT2 acylcoenzyme A: diacylglycerol acyltransferase family in the diatom Phaeodactylum tricornutum. FEBS Lett. 2013;587:481-7.

33. Niu YF, Zhang MH, Li DW, Yang WD, Liu JS, Bai WB, et al. Improvement of neutral lipid and polyunsaturated fatty acid biosynthesis by overexpressing a type 2 diacylglycerol acyltransferase in marine diatom Phaeodactylum tricornutum. Mar Drugs. 2013;11:4558-69.

34. Wagner M, Hoppe K, Czabany T, Heilmann M, Daum G, Feussner I, et al. Identification and characterization of an acyl-CoA:diacylglycerol acyltransferase 2 (DGAT2) gene from the microalga O. tauri. Plant Physiol Biochem. 2010;48:407-16.

35. Xu J, Kazachkov M, Jia Y, Zheng Z, Zou J. Expression of a type 2 diacylglycero acyltransferase from Thalassiosira pseudonana in yeast leads to incorporation of docosahexaenoic acid $\beta$-oxidation intermediates into triacylglycerol. FEBS J. 2013:280:6162-72.

36. Miller R, Wu G, Deshpande RR, Vieler A, Gartner K, Li X, et al. Changes in transcript abundance in Chlamydomonas reinhardtii following nitrogen deprivation predict diversion of metabolism. Plant Physiol. 2010;154:1737-52.

37. La Russa M, Bogen C, Uhmeyer A, Doebbe A, Filippone E, Kruse O, et al. Functional analysis of three type-2 DGAT homologue genes for triacylglycerol production in the green microalga Chlamydomonas reinhardtii. J Biotechnol. 2012;162:13-20.

38. Sanjaya MR, Durrett TP, Kosma DK, Lydic TA, Muthan B, et al. Altered lipid composition and enhanced nutritional value of Arabidopsis leaves following introduction of an algal diacylglycerol acyltransferase 2. Plant Cell. 2013;25: 677-93.

39. Boyle NR, Page MD, Liu B, Blaby IK, Casero D, Kropat J, et al. Three acyltransferases and nitrogen-responsive regulator are implicated in nitrogen starvation-induced triacylglycerol accumulation in Chlamydomonas. J Biol Chem. 2012;287:15811-25.

40. Chen JE, Smith AG. A look at diacylglycerol acyltransferases (DGATs) in algae. J Biotechnol. 2012;162:28-39.

41. Hofman K. Tmbase-a database of membrane spanning protein segments. Biol Chem Hoppe Seyler. 1993;374:166.

42. Hulo N, Bairoch A, Bulliard V, Cerutti L, De Castro E, Langendijk-Genevaux PS, et al. The PROSITE database. Nucleic Acids Res. 2006;34:227-30.

43. Jako C, Kumar A, Wei YD, Zou JT, Barton DL, Giblin EM, et al. Seed-specific over-expression of an Arabidopsis cDNA encoding a diacylglycerol acyltransferase enhances seed oil content and seed weight. Plant Physiol. 2001;126:861-74

44. Manas-Fernandez A, Vilches-Ferron M, Garrido-Cardenas JA, Belarbi EH, Alonso DL, Garcia-Maroto F. Cloning and molecular characterization of the
acyl-CoA: diacylglycerol acyltransferase 1 (DGAT1) gene from Echium. Lipids. 2009;44:555-68.

45. Lewin TM, Wang P, Coleman RA. Analysis of amino acid motifs diagnostic for the sn-glycerol-3-phosphate acyltransferase reaction. Biochemistry. 1999; 38:5764-71.

46. Joyce CW, Shelness GS, Davis MA, Lee RG, Skinner K, Anderson RA, et al. ACAT1 and ACAT2 membrane topology segregates a serine residue essential for activity to opposite sides of the endoplasmic reticulum membrane. Mol Biol Cell. 2000;11:3675-87.

47. Sandager L, Gustavsson MH, Stahl U, Dahlqvist A, Wiberg E, Banas A, et al. Storage lipid synthesis is non-essential in yeast. J Biol Chem. 2002;277:6478-82.

48. Zhang M, Fan JL, Taylor DC, Ohlrogge JB. DGAT1 and PDAT1 acyltransferases have overlapping functions in Arabidopsis triacylglycerol biosynthesis and are essential for normal pollen and seed development. Plant Cell. 2009;21:3885-901

49. IIIman AM, Scragg AH, Shales SW. Increase in Chlorella strains calorific values when grown in low nitrogen medium. Enzym Microb Technol. 2000;27: 631-5.

50. Li Y, Han F, Xu H, Mu J, Chen D, Feng B, et al. Potential lipid accumulation and growth characteristic of the green alga Chlorella with combination cultivation mode of nitrogen $(\mathrm{N})$ and phosphorus (P). Bioresour Technol. 2014;174:24-32.

51. Zhu S, Huang W, Xu J, Wang Z, Xu J, Yuan Z. Metabolic changes of starch and lipid triggered by nitrogen stanvation in the microalga Chlorella zofingiensis. Bioresour Technol. 2014;152:292-8.

52. Cakmak T, Angun P, Ozkan AD, Cakmak Z, Olmez TT, Tekinay T. Nitrogen and sulfur deprivation differentiate lipid accumulation targets of Chlamydomonas reinhardtii. Bioengineered. 2012;3:343-6.

53. Ho SH, Chen CY, Chang JS. Effect of light intensity and nitrogen starvation on $\mathrm{CO}_{2}$ fixation and lipid/carbohydrate production of an indigenous microalga Scenedesmus obliquus CNW-N. Bioresour Technol. 2012;113:244-52.

54. Jia J, Han D, Gerken HG, Li Y, Sommerfeld M, Hu Q, et al. Molecular mechanisms for photosynthetic carbon partitioning into storage neutral lipids in Nannochloropsis oceanica under nitrogen-depletion conditions. Algal Res. 2015;7:66-77.

55. Li J, Han D, Wang D, Ning K, Jia J, Wei L, et al. Choreography of transcriptomes and lipidomes of Nannochloropsis reveals the mechanisms of oil synthesis in microalgae. Plant Cell. 2014;26:1645-65.

56. Taylor DC, Zhang Y, Kumar A, Francis T, Giblin EM, Barton DL, et al. Molecular modification of triacylglycerol accumulation by over-expression of DGAT1 to produce canola with increased seed oil content under field conditions. Botany. 2009;87:533-43.

57. Appleyard RK. Segregation of new lysogenic types during growth of a doubly lysogenic strain derived from Escherichia Coli K12. Genetics. 1954;39:440-52

58. Elble R. A simple and efficient procedure for transformation of yeasts. Biotechniques. 1992;13:18-20.

59. Holsters M, de Waele D, Depicker A, Messens E, van Montagu M, Schell J. Transfection and transformation of Agrobacterium tumefaciens. Mol Gen Genet. 1978;163:181-7.

60. Clough SJ, Bent AF. Floral dip: a simplified method for Agrobacteriummediated transformation of Arabidopsis thaliana. Plant J. 1998;16:735-43.

61. Deblock M, Debrouwer D, Tenning P. Transformation of Brassica napus and Brassica oleracea using Agrobacterium tumefaciens and the expression of the bar and neo genes in the transgenic plants. Plant Physiol. 1989;91:694-701.

62. Jefferson RA, Kavanagh TA, Bevan MW. GUS fusions: $\beta$-glucuronidase as a sensitive and versatile gene fusion marker in higher plants. Embo J. 1987;6:3901-7.

63. Greenspan P, Mayer EP, Fowler SD. Nile Red - a selective fluorescent stain for intracellular lipid droplets. J Cell Biol. 1985;100:965-73.

64. Bligh EG, Dyer WJ. A rapid method of total lipid extraction and purification. Can J Biochem Physiol. 1959:37:911-7.

65. Katoh K, Toh H. Recent developments in the MAFFT multiple sequence alignment program. Brief Bioinform. 2008;9:286-98.

66. Price MN, Dehal PS, Arkin AP. FastTree 2- approximately maximum-likelihood trees for large alignments. Plos One. 2010;5:e9490.

67. Qi-Jun C, Hai-Meng Z, Jia C, Xue-Chen W. Using a modified TA cloning method to create entry clones. Anal Biochem. 2006;358:120-5. 
68. An G. High efficiency transformation of cultured tobacco cells. Plant Physiol. 1985;79:568-70.

69. Genschik P, Criqui MC, Parmentier Y, Derevier A, Fleck J. Cell cycledependent proteolysis in plants: identification of the destruction box pathway and metaphase arrest produced by the proteasome inhibitor MG132. Plant Cell. 1998;10:2063-76.

Submit your next manuscript to BioMed Central and we will help you at every step:

- We accept pre-submission inquiries

- Our selector tool helps you to find the most relevant journal

- We provide round the clock customer support

- Convenient online submission

- Thorough peer review

- Inclusion in PubMed and all major indexing services

- Maximum visibility for your research

Submit your manuscript at www.biomedcentral.com/submit 